\title{
Iterated Cesàro averages, frequencies of digits, and Baire category
}

by

J. Hyde (St Andrews), V. Laschos (Bath), L. Olsen (St Andrews), I. Petrykiewicz (St Andrews) and A. Shaw (St Andrews)

1. Statement of results. Fix a positive integer $N \geq 2$. Throughout this paper, we will consider the unique, non-terminating, base $N$ expansion of a number $x \in[0,1]$, written as

$$
x=\frac{d_{1}(x)}{N}+\frac{d_{2}(x)}{N^{2}}+\cdots+\frac{d_{n}(x)}{N^{n}}+\cdots \quad \text { with } d_{i}(x) \in\{0,1, \ldots, N-1\} .
$$

For each digit $i \in\{0,1, \ldots, N-1\}$, we will write

$$
\Pi_{i}(x ; n)=\frac{1}{n}\left|\left\{1 \leq j \leq n \mid d_{j}(x)=i\right\}\right|
$$

for the frequency of the digit $i$ among the first $n$ digits of $x$.

We recall that in a metric space $X$, a set $S$ is called residual if its complement is of the first category. Also recall that we say that a typical element $x$ has property $\mathrm{P}$ if the set $S=\{x \in X \mid x$ has property $\mathrm{P}\}$ is residual. We refer the reader to Oxtoby $[\mathrm{Ox}]$ for more details. The limiting behaviour of the frequencies $\Pi_{i}(x ; n)$ has been investigated extensively during the past many years. For example, Volkmann [Vo] and Šalát [Ša1] proved that for a typical $x$, we have

$$
\limsup _{n \rightarrow \infty} \Pi_{i}(x ; n)=1 \text { and } \quad \liminf _{n \rightarrow \infty} \Pi_{i}(x ; n)=0
$$

for all $0 \leq i \leq N-1$. Their proofs can also be found in various textbooks; see, for example, Billingsley [Bi, p. 16] and Hlawka [Hl, p. 77].

During the past 50 years these results have been extended and gen-

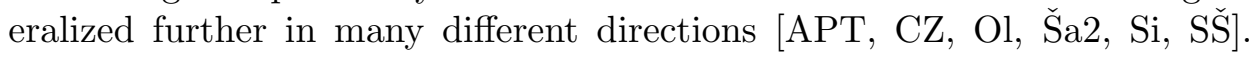
For example, recently Olsen [Ol] proved Theorem A below. To state this result we need to introduce some notation. Let $\Delta_{N}$ denote the family of

2010 Mathematics Subject Classification: 11K16, 11A63, 28A78.

Key words and phrases: Baire category, iterated Cesàro averages, iterated Cesàro means, frequencies of digits. 
$N$-dimensional probability vectors, i.e.

$$
\Delta_{N}=\left\{\left(p_{0}, p_{1}, \ldots, p_{N-1}\right) \mid p_{i} \geq 0, \sum_{i=0}^{N-1} p_{i}=1\right\},
$$

and write

$$
\boldsymbol{\Pi}(x ; n)=\left(\Pi_{0}(x ; n), \Pi_{1}(x ; n), \ldots, \Pi_{N-1}(x ; n)\right)
$$

for the vector of frequencies of digits. Then clearly $\boldsymbol{\Pi}(x ; n) \in \Delta_{N}$, and consequently the set of accumulation points of the sequence $(\boldsymbol{\Pi}(x ; n))_{n}$ is a subset of $\Delta_{N}$. Somewhat surprisingly, in [O1] it is proved that for a typical $x \in[0,1]$, the set of accumulation points of $(\boldsymbol{\Pi}(x ; n))_{n}$ is all of $\Delta_{N}$.

Theorem A $\mathrm{Ol}$. The set

$\left\{x \in[0,1] \mid\right.$ the set of accumulation points of $(\boldsymbol{\Pi}(x ; n))_{n=1}^{\infty}$ equals $\left.\Delta_{N}\right\}$ is residual.

Given a divergent sequence, forming its Cesàro averages may succeed in producing a convergent sequence, and one might expect that iterating this method would eventually give a convergent sequence when applied to the sequence $(\boldsymbol{\Pi}(x ; n))_{n}$. In this paper we will prove a somewhat unexpected result that strengthens Theorem A considerably. Namely, we show that for a typical $x$, the set of accumulation points of all higher order Cesàro averages of the sequence $(\boldsymbol{\Pi}(x ; n))_{n}$ equals $\Delta_{N}$. To state this result precisely, we make the following definitions. Let

$$
\Pi_{i}^{(1)}(x ; n)=\Pi_{i}(x ; n),
$$

and for $k \geq 2$, let

$$
\Pi_{i}^{(k)}(x ; n)=\frac{\sum_{j=1}^{n} \Pi_{j}^{(k-1)}(x ; j)}{n}
$$

denote $k$ th iterated Cesàro average. Next let

$$
\Pi^{(k)}(x ; n)=\left(\Pi_{0}^{(k)}(x ; n), \Pi_{1}^{(k)}(x ; n), \ldots, \Pi_{N-1}^{(k)}(x ; n)\right)
$$

denote the vector of $k$ th iterated Cesàro averages. Now we can state our main theorem.

THEOREM 1.1. The set

$$
\begin{gathered}
R=\left\{x \in[0,1] \mid \text { the set of accumulation points of }\left(\boldsymbol{\Pi}^{(k)}(x ; n)\right)_{n=1}^{\infty}\right. \\
\text { equals } \left.\Delta_{N} \text { for all } k \in \mathbb{N}\right\}
\end{gathered}
$$

is residual.

The proof of Theorem 1.1 is given in Section 2. Before presenting it, we make a few remarks concerning our result in different contexts. Below we will denote the Hausdorff dimension and the packing dimension by $\operatorname{dim}_{\mathrm{H}}$ 
and $\operatorname{dim}_{\mathrm{P}}$ respectively. The reader is referred to $\mathrm{Fa}$. for the definitions of dimensions.

Using Theorem 1.1 it is easy to find the packing dimension of the set $R$.

COROLlary 1.2. The packing dimension of the set $R$ equals 1 , i.e.

$$
\operatorname{dim}_{\mathrm{P}} R=1 \text {. }
$$

Proof. Recall that if $C$ is a compact subset of $\mathbb{R}$ and $M$ is a subset of $C$ with $\operatorname{dim}_{\mathrm{P}} M<\operatorname{dim}_{\mathrm{P}} C$, then $M$ is of the first category in $C$ (see [Ed, Exercise (1.8.4)]). Combined with Theorem 1.1, this implies that $\operatorname{dim}_{\mathrm{P}} R=$ $\operatorname{dim}_{P}([0,1])=1$.

Now we compare this with the Hausdorff dimension of $R$. It follows from OW] that $\operatorname{dim}_{\mathrm{H}} R=0$. Hence, in terms of dimensions, the size of $R$ varies between "very big" and "very small" depending on the exact viewpoint, but it follows from Theorem 1.1 that $R$ is always "very big" topologically.

This contrast between the topological and measure-theoretical viewpoints is also emphasized by Borel's Normal Number Theorem, which states that

$$
\Pi_{i}(x ; n) \rightarrow \frac{1}{N}
$$

for Lebesgue almost all $x \in[0,1]$. It follows from Borel's Normal Number Theorem that for Lebesgue almost all $x \in[0,1]$, the sequence $\left(\Pi_{i}(x ; n)\right)_{n}$ has only one accumulation point (namely $1 / N)$. This contrasts vastly with the topological point of view. Namely, Theorem 1.1 states that for a typical $x$, the set of accumulation points of $\left(\Pi_{i}^{(k)}(x ; n)\right)_{n}$ equals the simplex of $N$ dimensional probability vectors for all $k \in \mathbb{N}$.

2. Proof of Theorem 1.1. Throughout the proof, we will work with a subset of $[0,1]$, namely

$$
\mathbb{I}=[0,1] \backslash\{x \in[0,1] \mid x \text { has a terminating } N \text {-adic expansion }\} .
$$

To simplify the notation in our proof, we define the function $\varphi_{1}(x)=2^{x}$ and $\varphi_{m}(x)=\varphi_{1}\left(\varphi_{m-1}(x)\right)$ for $m \geq 2$. For brevity, write $\mathbb{D}=\left(\mathbb{Q}^{N} \cap \Delta_{N}\right) \backslash$ $\{(1,0,0, \ldots, 0)\}$. (We exclude one particular vector for technical reasons, which become apparent in the proof of Claim 2.) We define the property $\mathrm{P}$ as follows. We say that a sequence $\left(\boldsymbol{x}_{n}\right)_{n}$ in $\mathbb{R}^{N}$ has property $\mathrm{P}$ if for all $\boldsymbol{q} \in \mathbb{D}, m \in \mathbb{N}, i \in \mathbb{N}$, and $\epsilon>0$, there exists $j \in \mathbb{N}$ satisfying:

(i) $j \geq i$,

(ii) $j / 2^{j}<\epsilon$,

(iii) if $j<n<\varphi_{m}(j)$ then $\left|\boldsymbol{x}_{n}-\boldsymbol{q}\right|<\epsilon$.

Our proof of Theorem 1.1 will consist of three lemmas: 
(1) First we will prove that the set

$$
A=\left\{x \in \mathbb{I} \mid\left(\boldsymbol{\Pi}^{(1)}(x ; n)\right)_{n=1}^{\infty} \text { has property } \mathrm{P}\right\}
$$

is residual.

(2) Then we will show that if $\left(\boldsymbol{\Pi}^{(k)}(x ; n)\right)_{n=1}^{\infty}$ has property $\mathrm{P}$, then $\left(\boldsymbol{\Pi}^{(k+1)}(x ; n)\right)_{n=1}^{\infty}$ also has property P.

(3) Finally, we will show that $A \subseteq R$ (recall that $R$ is defined in Theorem 1.1).

Lemma 2.1. The set $A$ is residual (recall that $A$ is defined in (2.1)).

Proof. For fixed $h, m, i \in \mathbb{N}$ and $\boldsymbol{q} \in \mathbb{D}$, we define property $\mathrm{P}_{h, m, \boldsymbol{q}, i}$, as follows. We say that a sequence $\left(\boldsymbol{x}_{n}\right)_{n}$ has property $\mathrm{P}_{h, m, \boldsymbol{q}, i}$ if for every $\epsilon>1 / h$, there exists $j \in \mathbb{N}$ satisfying:

(i) $j \geq i$,

(ii) $j / 2^{j}<\epsilon$,

(iii) $j<n<\varphi_{m}\left(2^{j}\right) \Rightarrow\left|\boldsymbol{x}_{n}-\boldsymbol{q}\right|<\epsilon$.

Let $G_{h, m, \boldsymbol{q}, i}=\left\{x \in \mathbb{I} \mid\left(\boldsymbol{\Pi}^{(1)}(x ; n)\right)_{n=1}^{\infty}\right.$ has property $\left.\mathrm{P}_{h, m, \boldsymbol{q}, i}\right\}$. Clearly,

$$
\bigcap_{h \in \mathbb{N}} \bigcap_{m \in \mathbb{N}} \bigcap_{\boldsymbol{q} \in \mathbb{D}} \bigcap_{i \in \mathbb{N}} G_{h, m, \boldsymbol{q}, i}=A .
$$

Claim 1. $G_{h, m, \boldsymbol{q}, i}$ is open.

Proof. Let $x \in G_{h, m, \boldsymbol{q}, i}$. Since $x \in G_{h, m, \boldsymbol{q}, i}$, there exists a positive integer $j$ such that $j \geq i, j / 2^{j}<1 / h$, and if $j<n<\varphi_{m}\left(2^{j}\right)$, then $\left|\boldsymbol{\Pi}^{(1)}(x ; n)-\boldsymbol{q}\right|<$ $1 / h$.

We now choose $\delta$ to equal $1 / N^{\varphi_{m}\left(2^{j}\right)+1}$ if the $2^{j}$ th digit of $x$ is neither 0 nor $N-1$, otherwise we choose it to be $1 / N^{a}$ where $a$ is any integer such that the $(a-1)$ st and $(a-2)$ nd digits are not both either 0 or $N-1$, and that $a>\varphi_{m}\left(2^{j}\right)+1$. Then all $y \in B(x, \delta)$ have their first $\varphi_{m}\left(2^{j}\right)$ digits the same as $x$, and so $B(x, \delta) \subseteq G_{h, m, \boldsymbol{q}, i}$. This completes the proof of Claim 1 .

Claim 2. $G_{h, m, \boldsymbol{q}, i}$ is dense.

Proof. Let $x \in \mathbb{I}$ and $\delta>0$. We must now find $y \in B(x, \delta) \cap G_{h, m, \boldsymbol{q}, i}$. Let $t \in \mathbb{N}$ be such that $1 / N^{t}<\delta$. We can clearly choose a positive integer $s \in \mathbb{N}$ and $z_{1}, \ldots, z_{s} \in\{0,1, \ldots, N-1\}$ such that if $z=z_{1} / N+z_{2} / N^{2}+\cdots+z_{s} / N^{s}$ then $\boldsymbol{\Pi}^{(1)}(z ; s)=\boldsymbol{q}$. Let

$$
y=\frac{d_{1}(x)}{N}+\cdots+\frac{d_{t}(x)}{N^{t}}+\sum_{i=0}^{\infty}\left(\frac{z_{1}}{N^{t+i s+1}}+\frac{z_{2}}{N^{t+i s+2}}+\cdots+\frac{z_{s}}{N^{t+i s+s}}\right) .
$$

Then $y \in B(x, \delta)$ (as $y$ has the first $t$ digits the same as $x$ ).

Next we show that $y \in G_{h, m, \boldsymbol{q}, i}$. All $z_{i}$ 's cannot be 0 , because we excluded the vector $(1,0, \ldots, 0)$. Therefore, $y$ has a non-terminating $N$-adic 
expansion. Let $\epsilon \geq 1 / h$, and choose $j$ such that

$\frac{j}{2^{j}}<\epsilon \quad$ and $\quad j \geq N \max \left(\max _{l \in\{0,1, \ldots, N-1\}}\left|\frac{N_{l}(z ; s)(2+t / s)+N_{l}(y ; t)}{\epsilon}\right|, i, t\right)$

where $N_{l}(x ; n)=\left|\left\{0<j \leq n \mid d_{j}(x)=l\right\}\right|$. Fix a positive integer $n$ with $j<n<\varphi_{m}\left(2^{j}\right)$ and observe that we can find integers $r$ and $b$, with $0 \leq r<s$ and $0 \leq b<N_{l}(z ; s)$, such that $n=t+\lfloor(n-t) / s\rfloor s+r$, and $N_{l}(y ; n)=N_{l}(y ; t)+\lfloor(n-t) / s\rfloor N_{l}(z ; s)+b$. We now have

$$
\begin{aligned}
& \left|\boldsymbol{\Pi}^{(1)}(y ; n)-\boldsymbol{q}\right|=N \max _{l}\left|\Pi_{l}^{(1)}(y ; n)-\frac{N_{l}(z ; s)}{s}\right| \\
& =N \max _{l}\left|\frac{N_{l}(y ; n)}{n}-\frac{n \frac{N_{l}(z ; s)}{s} \mid}{n}\right| \\
& =N \max _{l}\left|\frac{N_{l}(y ; t)+\left\lfloor\frac{n-t}{s}\right\rfloor N_{l}(z ; s)+b}{n}-\frac{\left(t+\left\lfloor\frac{n-t}{s}\right\rfloor s+r\right) \frac{N_{l}(z ; s)}{s} \mid}{n}\right| \\
& \leq N \max _{l}\left(\left\lfloor\frac{\left\lfloor\frac{n-t}{s}\right\rfloor N_{l}(z ; s)-\frac{N_{l}(z ; s)}{s}(t+r)-N_{l}(z ; s)\left\lfloor\frac{n-t}{s}\right\rfloor}{n} \mid+\frac{N_{l}(y ; t)+b}{n}\right)\right. \\
& \leq N \max _{l}\left(\left\lfloor\frac{\left.-\frac{N_{l}(z ; s)}{s}(t+r)\right\rfloor}{n} \mid+\frac{N_{l}(y ; t)+N_{l}(z ; s)}{n}\right)\right. \\
& \leq N \max _{l}\left(\frac{\frac{N_{l}(z ; s)}{s}(t+r)+N_{l}(y ; t)+N_{l}(z ; s)}{n}\right) \\
& \leq N \max _{l}\left(\frac{\frac{N_{l}(z ; s)}{s}(t+s)+N_{l}(y ; t)+N_{l}(z ; s)}{j}\right) \\
& \leq N \max _{l}\left(\frac{N_{l}(z ; s)(2+t / s)+N_{l}(y ; t)}{j}\right) \leq \epsilon,
\end{aligned}
$$

where the maximum is over $l \in\{0,1, \ldots, N-1\}$. This shows that $y \in$ $G_{h, m, \boldsymbol{q}, i}$, and completes the proof of Claim 2 .

It follows from Claims 1 and 2 that $A$ is the countable intersection of open and dense sets, and hence residual. This completes the proof of Lemma 2.1.

Lemma 2.2. If $\left(\boldsymbol{\Pi}^{(k)}(x ; n)\right)_{n=1}^{\infty}$ has property $\mathrm{P}$, then $\left(\boldsymbol{\Pi}^{(k+1)}(x ; n)\right)_{n=1}^{\infty}$ also has property $\mathrm{P}$.

Proof. Let $\left(\boldsymbol{\Pi}^{(k)}(x ; n)\right)_{n=1}^{\infty}$ have property $\mathrm{P}$, and fix $\epsilon>0, \boldsymbol{q} \in \mathbb{D}, i \in \mathbb{N}$ and $m \in \mathbb{N}$. Since $\left(\boldsymbol{\Pi}^{(k)}(x ; n)\right)_{n=1}^{\infty}$ has property P, there exists $j^{\prime} \in \mathbb{N}$ with $j^{\prime} \geq i, j^{\prime} / 2^{j^{\prime}}<\epsilon / 3$, and such that if $j^{\prime}<n<\varphi_{m+1}\left(2^{j^{\prime}}\right)$ then $\left|\boldsymbol{\Pi}^{(k)}(x ; n)-\boldsymbol{q}\right|$ $<\epsilon / 3$. Let $j=2^{j^{\prime}}$. For all $j<n<\varphi_{m}\left(2^{j}\right)$ (i.e. $2^{j^{\prime}}<n<\varphi_{m+1}\left(2^{j^{\prime}}\right)$ ), we 
have

$$
\begin{aligned}
\left|\boldsymbol{\Pi}^{(k+1)}(x ; n)-\boldsymbol{q}\right|= & \left|\frac{\boldsymbol{\Pi}^{(k)}(x ; 1)+\boldsymbol{\Pi}^{(k)}(x ; 2)+\cdots+\boldsymbol{\Pi}^{(k)}(x ; n)}{n}-\boldsymbol{q}\right| \\
= & \mid \frac{\boldsymbol{\Pi}^{(k)}(x ; 1)+\cdots+\boldsymbol{\Pi}^{(k)}\left(x ; j^{\prime}\right)}{n} \\
& +\frac{\boldsymbol{\Pi}^{(k)}\left(x ; j^{\prime}+1\right)+\cdots+\boldsymbol{\Pi}^{(k)}(x ; n)-\left(n-j^{\prime}\right) \boldsymbol{q}}{n}-\frac{j^{\prime} \boldsymbol{q}}{n} \mid \\
\leq & \frac{\left|\boldsymbol{\Pi}^{(k)}(x ; 1)+\cdots+\boldsymbol{\Pi}^{(k)}\left(x ; j^{\prime}\right)\right|}{n} \\
& +\frac{\left|\boldsymbol{\Pi}^{(k)}\left(x ; j^{\prime}+1\right)-\boldsymbol{q}\right|+\cdots+\left|\boldsymbol{\Pi}^{(k)}(x ; n)-\boldsymbol{q}\right|}{n}+\frac{\left|j^{\prime} \boldsymbol{q}\right|}{n} \\
\leq & \frac{j^{\prime}}{n}+\frac{\epsilon}{3} \frac{n-j^{\prime}}{n}+\frac{j^{\prime}}{n} \leq \frac{j^{\prime}}{2^{j^{\prime}}}+\frac{\epsilon}{3}+\frac{j^{\prime}}{2^{j^{\prime}}} \leq \frac{\epsilon}{3}+\frac{\epsilon}{3}+\frac{\epsilon}{3}=\epsilon .
\end{aligned}
$$

This completes the proof of Lemma 2.2.

Lemma 2.3. The set $A$ is a subset of $R$ (recall that $R$ is defined in Theorem 1.1).

Proof. Let $x \in A$. By Lemma 2.2, we deduce that $\left(\boldsymbol{\Pi}^{(k)}(x ; n)\right)_{n}$ has property $\mathrm{P}$ for all $k$. We now want to show that $x \in R$, i.e. the set of accumulation points of $\left(\boldsymbol{\Pi}^{(k)}(x ; n)\right)_{n}$ equals $\Delta_{N}$. It is clear that the set of accumulation points of $\left(\boldsymbol{\Pi}^{(k)}(x ; n)\right)_{n}$ is a subset of $\Delta_{N}$. Hence, it suffices to show that each $\boldsymbol{p} \in \Delta_{N}$ is an accumulation point of $\left(\boldsymbol{\Pi}^{(k)}(x ; n)\right)_{n}$. Therefore, let $\boldsymbol{p} \in \Delta_{N}$. Fix $l \in \mathbb{N}$ and $\boldsymbol{q} \in \mathbb{D}$ such that $|\boldsymbol{p}-\boldsymbol{q}| \leq 1 / l$.

We first observe that we can find $n_{l}>l$ such that

$$
\left|\boldsymbol{q}-\boldsymbol{\Pi}^{(k)}\left(x ; n_{l}\right)\right| \leq 1 / l .
$$

We now prove (2.2). Indeed, since $x \in A$, we conclude from Lemma 2.2 that $\left(\boldsymbol{\Pi}^{(k)}(x ; n)\right)_{n}$ has property P. In particular, we can find $j \in \mathbb{N}$ with $l \leq j$ and such that if $j<n<\varphi_{m}\left(2^{j}\right)$ then $\left|\boldsymbol{\Pi}^{(k)}(x ; n)-\boldsymbol{q}\right|<1 / l$. Hence if $n_{l}$ is any integer with $j<n_{l}<\varphi_{m}\left(2^{j}\right)$ then $\left|\boldsymbol{\Pi}^{(k)}\left(x ; n_{l}\right)-\boldsymbol{q}\right|<1 / l$.

Hence, the sequence $\left(n_{l}\right)_{l}$ satisfies $n_{l}$ and

$$
\left|\boldsymbol{p}-\boldsymbol{\Pi}^{(k)}\left(x ; n_{l}\right)\right| \leq|\boldsymbol{p}-\boldsymbol{q}|+\left|\boldsymbol{\Pi}^{(k)}\left(x ; n_{l}\right)-\boldsymbol{q}\right| \leq 2 / l .
$$

Since $n_{l}>l$, we can extract an increasing subsequence $\left(n_{l_{u}}\right)_{u}$ of $\left(n_{l}\right)_{l}$. It now follows from $(2.3)$ that $\boldsymbol{\Pi}^{(k)}\left(x ; n_{l_{u}}\right) \rightarrow \boldsymbol{p}$. Hence $\boldsymbol{p}$ is an accumulation point of $\left(\boldsymbol{\Pi}^{(k)}\left(x ; n_{l_{u}}\right)\right)_{u=1}^{\infty}$. This completes the proof of Lemma 2.3.

Proof of Theorem 1.1. It follows from Lemma 2.3 that $A \subseteq R$. By Lemma $2.1, A$ is residual in $\mathbb{I}$. Since it is easily seen that $[0,1] \backslash \mathbb{I}$ is a countable union of nowhere dense sets, $A$ is residual in $[0,1]$. Hence, we conclude that $R$ is residual. 
Acknowledgments. The fifth-named author is supported by the Carnegie Trust.

\section{References}

[APT] S. Albeverio, M. Pratsiovytyi and G. Torbin, Topological and fractal properties of subsets of real numbers which are not normal, Bull. Sci. Math. 129 (2005), 615-630.

[Bi] P. Billingsley, Probability and Measure, 2nd ed., Wiley, New York, 1986.

[CZ] C. Calude and T. Zamfirescu, Most numbers obey no probability laws, in: Automata and Formal Languages, VIII (Salgótarján, 1996), Publ. Math. Debrecen 54 (1999), suppl., 619-623.

[Ed] G. A. Edgar, Integral, Probability, and Fractal Measures, Springer, New York, 1998.

[Fa] K. J. Falconer, Fractal Geometry, Wiley, 1990.

[Hl] E. Hlawka, Theorie der Gleichverteilung, Bibliographisches Institut Wissenschaftsverlag, Mannheim, 1979.

[Ol] L. Olsen, Extremely non-normal numbers, Math. Proc. Cambridge Philos. Soc. 137 (2004), 43-53.

[OW] L. Olsen and S. Winter, Normal and non-normal points of self-similar sets and divergence points of self-similar measures, J. London Math. Soc. 67 (2003), 103122 .

[Ox] J. C. Oxtoby, Measure and Category, 2nd ed., Springer, New York, 1996.

[Ša1] T. Šalát, A remark on normal numbers, Rev. Roumaine Math. Pures Appl. 11 (1966), 53-56.

[Ša2] -, Über die Cantorschen Reihen, Czechoslovak Math. J. 18 (93) (1968), 25-56.

[SŠ] F. Schweiger and T. Šalát, Some sets of sequences of positive integers and normal numbers, Rev. Roumaine Math. Pures Appl. 26 (1981), 1255-1264.

[Si] K. Sigmund, Nombres normaux et théorie ergodique, in: Théorie ergodique (Actes Journées Ergodiques, Rennes, 1973/1974), Lecture Notes in Math. 532, Springer, Berlin, 1976, 202-215.

[Vo] B. Volkmann, Gewinnmengen, Arch. Math. (Basel) 10 (1959), 235-240.

J. Hyde, L. Olsen, I. Petrykiewicz, A. Shaw

Department of Mathematics

University of St Andrews

St Andrews, Fife KY16 9SS, Scotland

E-mail: jth4@st-and.ac.uk

V. Laschos

Department of Mathematics University of Bath

Bath, BA2 7AY, England lo@st-and.ac.uk ip46@st-and.ac.uk afs8@st-and.ac.uk

Received on 14.9.2009

and in revised form on 14.1.2010 